Research Paper

\title{
Bone Windows for Distinguishing Malignant from Benign Primary Bone Tumors on FDG PET/CT
}

\author{
Colleen M. Costelloe ${ }^{\circledR}$, Hubert H. Chuang'2, Beth A. Chasen², Tinsu Pan³, Patricia S. Fox ${ }^{4}$, Roland L. Bas- \\ sett $^{4}$, and John E. Madewell ${ }^{1}$
}

1. Department of Diagnostic Radiology,

2. Department of Nuclear Medicine,

3. Department of Imaging Physics, and

4. Department of Biostatics, UT MD Anderson Cancer Center, 1515 Holcombe Blvd., Houston, TX 77030, USA

\begin{abstract}
$\triangle$ Corresponding author: Colleen M. Costelloe, MD, UT MD Anderson Cancer Center, 1400 Pressler St., Unit 1475, Houston, TX 77030. Phone: 713-563-1260

() Ivyspring International Publisher. This is an open-access article distributed under the terms of the Creative Commons License (http://creativecommons.org/ licenses/by-nc-nd/3.0/). Reproduction is permitted for personal, noncommercial use, provided that the article is in whole, unmodified, and properly cited.
\end{abstract}

Received: 2013.03.16; Accepted: 2013.05.02; Published: 2013.08.09

\begin{abstract}
Objective. The default window setting on PET/CT workstations is soft tissue. This study investigates whether bone windowing and hybrid FDG PET/CT can help differentiate between malignant and benign primary bone tumors.

Materials and methods. A database review included 98 patients with malignant $(n=64)$ or benign primary bone $(n=34)$ tumors. The reference standard was biopsy for malignancies and biopsy or >I year imaging follow-up of benign tumors. Three radiologists and/or nuclear medicine physicians blinded to diagnosis and other imaging viewed the lesions on $\mathrm{CT}$ with bone windows (CT-BW) without and then with PET (PET/CT-BW), and separate PET-only images for malignancy or benignity. Three weeks later the tumors were viewed on $C T$ with soft tissue windows (CT-STW) without and then with PET (PET/CT-STW).

Results. Mean sensitivity and specificity for identifying malignancies included: CT-BW: $96 \%, 90 \%$; CT-STW: 90\%, 90\%; PET/CT-BW: 95\%, 85\%, PET/CT-STW: 95\%, 86\% and PET-only: 96\%, 75\%, respectively. CT-BW demonstrated higher specificity than PET-only and PET/CT-BW ( $\mathrm{P}=0.0005$ and $p=0.0103$, respectively) and trended toward higher sensitivity than CT-STW $(p=0.0759)$. Malignant primary bone tumors were more avid than benign lesions overall $(p<0.000 I)$ but the avidity of benign aggressive lesions (giant cell tumors and Langerhans Cell Histiocytosis) trended higher than the malignancies $(p=0.08)$.

Conclusion. Bone windows provided high specificity for distinguishing between malignant and benign primary bone tumors and are recommended when viewing FDG PET/CT.
\end{abstract}

Key words: FDG PET/CT; CT; primary bone tumor; bone window; soft tissue window.

\section{Introduction}

Early in the history of CT scanning, specialized windowing was found to be beneficial for the analysis of bone lesions. Becker et al [1] demonstrated in 1978 that more calvarial metastases were detected with bone windows than with brain windows. The human eye distinguishes a limited number of shades of grey and the widely varying densities of body structures cannot be seen equally well on a single computer setting. Each CT window algorithm focuses the grey scale in an appropriate manner to enhance anatomy of a selected density. On the workstations used to view the PET/CT scans in this study (GE Advantage Win- 
dows version 4.3), bone windows have CT unit settings with a relatively high level of 350 and a wide width of 2000. The high level is suitable for evaluating dense structures such as bone, and the wide width encompasses a large range of tissue densities. Lung windows have a low level of -496 that is suitable for evaluating air-containing structures, and also have a wide width (1450). Soft tissue windows are leveled near water (40) with a narrow width of 400 that enhances the subtle differences in soft tissue structures which typically have a density close to water [2].

Bone windows can aid in the interpretation of CT scans in numerous capacities, such as through the detection of aggressive features caused by osteomyelitis [3] and bone tumors [4]. In a study of stenoclavicular osteomyelitis that included both bone and soft tissue windows, the bone windows were found to be more sensitive for detecting small erosions and periostitis [3]. The detection of osteolysis or periosteal reaction can facilitate biopsy or aspiration of aggressive processes in order to determine etiology and allow treatment planning.

The use of bone windows has been shown to increase the detection of bone metastases [5], and improve the efficacy of diagnosis for bone lesions by allowing differentiation between metastases and degenerative change [6]. Bone windows can be more efficacious than soft tissue windows for identifying distinctive features of primary bone tumors such as the nidus of osteoid osteomas [7], and many investigators have used bone window settings as a matter of course for evaluating other primary bone tumors [8-11].

The standard default CT window setting on positron emission tomography (PET) workstations is soft tissue. The purpose of this study was to evaluate whether bone windowing can aid in differentiating between malignant and benign primary bone tumors on FDG PET/CT, justifying the extra time and effort needed to view the lesions in this manner. PET/CT was chosen for this analysis in order to also test the conclusions of the small number of prior investigations that have demonstrated that the addition of CT to PET is beneficial for evaluating primary bone tumors.

\section{Materials and Methods}

\section{Patient Selection}

The study was performed in compliance with the Health Insurance Portability and Accountability Act. Approval was obtained from the institutional review board. A keyword search of the institutional database was performed from January 1, 2007 - October 1, 2010.
Keywords included PET and bone island, osteoma, osteoblastoma, osteoid osteoma, fibrous dysplasia, nonossifying fibroma, ossifying fibroma, fibrous cortical defect, osteofibrous dysplasia, osteochondroma, enchondroma, chondroblastoma, chondromyxoid fibroma, cortical desmoid, desmoplastic fibroma, intraosseous lipoma, glomus tumor, hemangioma, bone cyst, giant cell tumor, giant cell reparative granuloma, eosinophilic granuloma/Langerhans cell histiocytosis, chordoma, osteosarcoma, fibrosarcoma, chondrosarcoma, Ewing sarcoma and adamantinoma. Excluded were patients who received therapy for bone malignancy prior to FDG PET/CT, lesions not visible on the $\mathrm{CT}$, below $8 \mathrm{~mm}$ in size or with no FDG uptake, unbiopsied malignancies or unbiopsied benign lesions with $<1$ year imaging follow-up, patients with bone or FDG-avid metastases, and lesions distorted by prior biopsy or metal artifact. One unbiopsied chondrosarcoma in a patient with Ollier's disease was included on the basis of short-interval enlargement of the lesion and increasing frequency of pain. The patient was lost to follow-up prior to recommended hemipelvectomy. All included scans were performed at our institution.

\section{Image Review}

One radiologist with 7 years of experience with FDG PET/CT, one nuclear medicine physician with 3 years of experience with FDG PET/CT, and one physician who was dual board certified in radiology and nuclear medicine with 6 years of experience reviewed the images. Each tumor was evaluated on the CT portion of the scan and a diagnosis of malignant or benign was determined on bone windows (CT-BW). The diagnosis was then re-considered with the addition of PET (PET/CT-BW). Because the emphasis of this part of the study was on CT windowing and not PET, there was no hiatus between $\mathrm{CT}$ readings without and with PET. Three weeks later [12], the process was repeated with soft tissue windows (CT-STW, PET/CT-STW). The nuclear medicine physicians also evaluated PET-only images for diagnosis of malignancy or benignity with no CT data. Due to lack of identifying anatomic landmarks on PET-only images, no hiatus was required. The readers were blinded to diagnosis, medical history and all other imaging studies. All images were viewed on GE Advantage Windows (AW) workstations, version 4.3.

\section{PET/CT}

Integrated PET/CT systems were utilized to acquire imaging data (Discovery ST, STe, RX, or VCT) General Electric Healthcare, Milwaukee, WI). Whole-body examinations were performed from the 
level of the vertex of the skull or orbits through the upper thighs or lower legs/toes. PET/CT was performed in accordance with guidelines published by the National Cancer Institute [13]. All patients were fasted for a minimum of 6 hours with blood glucose of $80-120 \mathrm{mg} / \mathrm{dL}$ (4.4-6.6 mmol/L) prior to intravenous administration of approximately $10 \mathrm{mCi}(370 \mathrm{MBq})$ of FDG for 3D and 15-20 mCi (555-740 MBq) for 2D acquisition. Unenhanced CT was used for attenuation correction and diagnosis and it included $3.75 \mathrm{~mm}$ axial slice placement with $3.27 \mathrm{~mm}$ slice spacing, $120 \mathrm{kV}$, $300 \mathrm{~mA}$ and $0.5 \mathrm{~s}$ gantry rotation at $55 \mathrm{~mm} / \mathrm{s}$ table speed. Emission PET was performed 60 minutes after FDG administration at 3 minutes per bed station.

\section{Statistical Analysis}

Generalized estimating equations were used to estimate sensitivity and specificity taking into account multiple readings per patient, reader effect, window effect, and SUVmax. The Tukey- Kramer adjustment was used to account for pairwise comparisons between windows. Wilcoxon rank sum tests were used to compare SUVmax by subtypes with p-values computed using the normal approximation. All statistical analyses were performed using SAS 9.3 for Windows (Copyright $@ 2011$ by SAS Institute Inc., Cary, NC).

\section{Results}

The keyword search returned 6972 scans. The search found no FDG PET/CT scans with osteofibrous dysplasias, chondromyxoid fibromas, desmoplastic fibromas, ossifying fibromas, adamantinomas or glomus tumors. No scans meeting the inclusion criteria were found for osteoblastomas, chondroblastomas, cortical desmoids, osteomas or bone cysts. Review of bone islands and hemangiomas were truncated at 50 each due to lack of FDG avid lesions. No spinal hemangiomas were included. One biopsy-proven, expansile hemangioma of the rib, whose differential diagnosis did not include hemangioma and was not found under the hemangioma keyword search, was included. The terms "nonossifying fibroma" and "fibroxanthoma" are used interchangeably

A total of 98 tumors were included in the study. They included osteosarcoma $(n=47)$, Ewing sarcoma $(n=10)$, chondrosarcoma $(n=5)$, high grade unclassified sarcoma of bone $(n=1)$, chordoma $(n=1)$, giant cell tumor of bone $(n=1)$, giant cell reparative granuloma $(\mathrm{n}=1)$, Langerhans Cell Histiocytosis $(\mathrm{LCH}, \mathrm{n}=1)$, fibrous dysplasia $(n=10)$, enchondroma $(n=13)$, osteochondroma $(n=3)$, nonossifying fibroma $(n=1)$, fibrous cortical defect $(n=2)$, hemangioma $(n=1)$, intraosseous lipoma $(n=1)$.
Malignant primary bone tumors were more avid than benign lesions overall ( $p<0.0001$, Table 1, Fig. 1). The mean and range of SUVmax for malignancies was $12.6(2.2$ - 56.9) and for benign lesions was 4.3 (1.2 21.1). Per group, chondrosarcomas and Ewing sarcomas were not significantly different from each other in FDG avidity $(p=0.81)$ and were significantly less avid than osteosarcomas $(p=0.02)$. Chondrosarcomas $(8.8$, 3.9-20.5) were significantly more avid than the benign cartilage tumors (enchondromas and osteochondromas, 2.21,1.20-3.60, $p=0.001)$. Giant cell tumors were significantly more avid than chondrosarcoma/Ewing sarcomas $(p=0.04)$ and not significantly different from the osteosarcomas $(p=0.14)$. The benign aggressive lesions (giant cell tumors and LCH; 17.63,14.10-21.10) were significantly more avid than other benign tumors overall $(p=0.0062)$ and trended toward greater avidity than the malignancies overall (0.08). The avidity of fibrous dysplasias trended higher than other benign lesions $(p=0.09)$ but was significantly lower than the malignancies $(\mathrm{p}<0.0001)$.

Mean and range of sensitivity and specificity values for distinguishing between malignant and benign primary bone tumors are listed in Table 2. All significant findings for sensitivity and specificity include the following: CT-BW demonstrated higher specificity than PET-only and PET/CT-BW $(\mathrm{p}=0.0005$ and $\mathrm{p}=0.0103$, respectively). CT-BW also trended toward higher sensitivity than CT-STW $(\mathrm{p}=0.0759)$. PET/CT-STW trended toward higher sensitivity than CT- STW $(p=0.0662)$ and a higher specificity than PET-only $(\mathrm{p}=0.0859)$.

Table I. FDG Avidity of Primary Bone Tumors.

\begin{tabular}{lll}
\hline Tumor & SUVmax & $\begin{array}{l}\text { Number of } \\
\text { Tumors }\end{array}$ \\
\hline Unclassified Sarcoma & 27.8 & 1 \\
Giant Cell Tumors & $19.4(17.7-21.1)$ & 2 \\
Langerhans Cell Histiocytosis & 14.1 & 1 \\
Osteosarcoma & $13.7(3.8-56.9)$ & 47 \\
Chondrosarcoma & $8.8(3.9-20.5)$ & 5 \\
Ewing sarcoma & $8.4(2.2-15.1)$ & 10 \\
Chordoma & 6.1 & 1 \\
Fibrous Dysplasia & $4.4(2.2-15.7)$ & 10 \\
Nonossifying Fibroma & 3.9 & 1 \\
Fibrous Cortical Defect & $3.4(2.5-4.3)$ & 2 \\
Hemangioma & 2.7 & 1 \\
Osteochondroma & $2.6(1.9-3.6)$ & 3 \\
Enchondroma & $2.1(1.2-3.3)$ & 13 \\
Intraosseous Lipoma & 1.2 & 1 \\
\hline
\end{tabular}




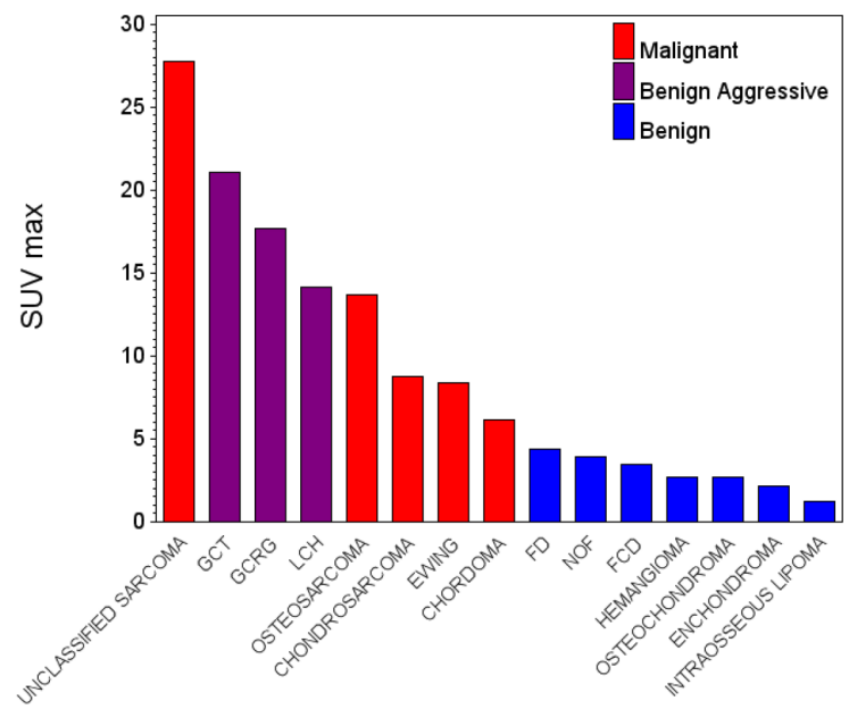

Tumor Type

Fig I. GCT - Giant cell tumor of bone. GCRG - Giant cell reparative granuloma, LCH - Langerhans Cell Histiocytosis, FD - Fibrous Dysplasia, NOF - Nonossifying fibroma/fibroxanthoma, FCD - Fibrous cortical defect.

Table 2. Sensitivity and Specificity.

\begin{tabular}{lll}
\hline Dataset & $\begin{array}{l}\text { Sensitivity }(\%) \\
\text { mean (range) }\end{array}$ & $\begin{array}{l}\text { Specificity }(\%) \\
\text { mean (range) }\end{array}$ \\
\hline CT-BW & $96(95-97)$ & $90(88-91)$ \\
PET/CT-BW & $95(94-97)$ & $85(79-88)$ \\
CT-STW & $90(86-94)$ & $90(88-91)$ \\
PET/CT-STW & $95(94-95)$ & $86(85-88)$ \\
PET-only & $96(94-98)$ & $75(68-82)$ \\
\hline
\end{tabular}

\section{Discussion}

Prior studies have attempted to differentiate malignant from benign bone lesions on the basis of FDG uptake alone, prior to the development and widespread usage of dual-modality, hybrid PET/CT scanners [14-17]. This effort was not fully successful because high FDG avidity was found in several benign histologies which overlapped with the avidity of the malignant lesions. Our study found that the FDG uptake of the benign aggressive lesions (giant cell tumors and LCH) trended higher than that of the malignancies as a whole $(p=0.08)$. It has been suggested that giant cells and histiocytic cells may be responsible for high uptake within many benign primary bone tumors [15]. All of the benign lesions with high uptake in our study contained a preponderance of either giant cells (giant cell tumor of bone, giant cell reparative granuloma) or histiocytic cells (LCH).
We have shown a higher overall mean avidity in osteosarcomas as compared to Ewing sarcomas [17] and similar mean avidity when comparing Ewing sarcomas and chondrosarcomas. Chondrosarcomas can be difficult to distinguish from benign cartilage lesions on conventional imaging modalities such as radiographs, CT, MRI and skeletal scintigraphy, as well as on histopathologic analysis.

Chondrosarcomas (particularly low grade lesions) have been reported as having a predilection for low FDG uptake in comparison with other primary bone malignancies [17-20] which could also be confounding. Factors that may contribute to the lower avidity include a high proportion of acellular gelatinous matrix with respect to cellular density and lower mitotic rates than higher grade tumors. Dedicated analysis of cartilage lesions as a whole has previously demonstrated the FDG avidity of malignancies to be significantly higher than benign lesions [18, 21]. Our study showed that the average FDG uptake of chondrosarcomas was as high as Ewing sarcoma though lower than osteosarcoma. The most avid cartilage lesion was a mesenchymal chondrosarcoma $(n=1)$ with an SUVmax of 20.5 (other lesions included grade 1, 2A, 2/3 and unbiopsied - MD Anderson grading system of chondrosarcomas). Excluding the mesenchymal chondrosarcoma, the mean SUVmax of the chondrosarcomas decreased from 8.8 to 6.4 (3.9-9.2). The adjusted value remains higher than the mean SUVmax of the benign cartilage lesions (2.2) and the benign lesions overall (4.3). While the SUVmax of the most avid benign cartilage lesion (3.6, osteochondro$\mathrm{ma}$ ) approached that of the least avid chondrosarcoma $(3.9$, grade 1$)$, we agree with prior studies that suggest a potential diagnostic advantage with the use of FDG PET for distinguishing malignant from benign cartilage lesions [18, 21].

Unlike other reported data $[14-15,17,22]$ the mean FDG uptake of the benign fibrous lesions in our study did not approach the mean avidity of the malignancies. This may have been due to our small sample size.

Chordoma, a relatively indolent malignancy with a strong tendency for local recurrence, demonstrated FDG uptake between the mean of the least avid malignancy (Ewing-sarcoma) and the most avid benign lesion (fibrous dysplasia). Our results suggest that the FDG uptake across all of the tumors in our sample corresponds better with the locally destructive than the metastatic potential of the lesions (Fig. 1).

With the advent of hybrid PET/CT scanners, one prior study has systematically assessed the value of the added CT portion of the examination, and has shown that the $\mathrm{CT}$ is beneficial for identifying malig- 
nant primary bone tumors [19 ]. Published sensitivity and specificity data from that study were $91 \%$ and $77 \%$, respectively [19]. Our mean sensitivity and specificity were $95 \%$ and $86 \%$, respectively. Our mean sensitivity corresponds to the published number and our specificity is higher. This may have been due to our study design that mandated an in-depth review of the CT prior to evaluating the PET dataset.

We have conducted the first study to separate CT from PET/CT, and to examine the effect of windowing in the context of primary bone tumors. Our unique mean sensitivity and specificity data include CT-BW (96\%, 90\%), CT-STW (90\%, 90\%) PET/CT-BW (95\%, $85 \%)$, and PET/CT-STW (95\%, 86\%).

Specificity for the detection of primary bone tumors was significantly high with CT-BW when compared to PET/CT-BW ( $p=0.0103)$. This suggests that the fine bony detail that is visible with CT-BW is useful for distinguishing benign from malignant lesions, and that this bony detail may be obscured by FDG uptake. Specificity was also higher with CT-BW than with PET-only $(p=0.0005)$, indicating that the anatomic data provided by the bone windows was more useful for identifying the lesions than FDG-uptake alone (Fig. 2). Sensitivity for detecting malignancy trended higher with CT-BW than CT-STW ( $p=0.0759$, Fig $3 \mathbf{A}$ and $\mathbf{D}$ ). The margins of primary bone tumors are an important indicator of their biological potential [24-25]. Malignancies are more likely to produce a greater degree of osteolysis than benign lesions [24-25]. Soft tissue windows may over-emphasize tumor margins and spuriously make cortical or trabecular bone appear intact. Bone windows can reveal the osteolysis that is obscured by the soft tissue window setting (Fig. 4).

The emphasis that our study placed on CT was considered justified given the inability of priorPET-only investigations to reliably distinguish between malignant and benign bone lesions [14-15]. We recommend the use of CT-only bone windows when evaluating bone tumors on FDG PET/CT. Radiographs were not evaluated by this study. Radiographs are recommended as the first step in the imaging diagnosis of bone lesions [24, 26]. CT with bone windowing is immediately available and can be used as an adjunct to radiography.

In conclusion, our study found high specificity for distinguishing between benign and malignant primary bone tumors using bone window settings and it is recommended that bone windows be used for evaluating osseous tumors on FDG PET/CT scans.

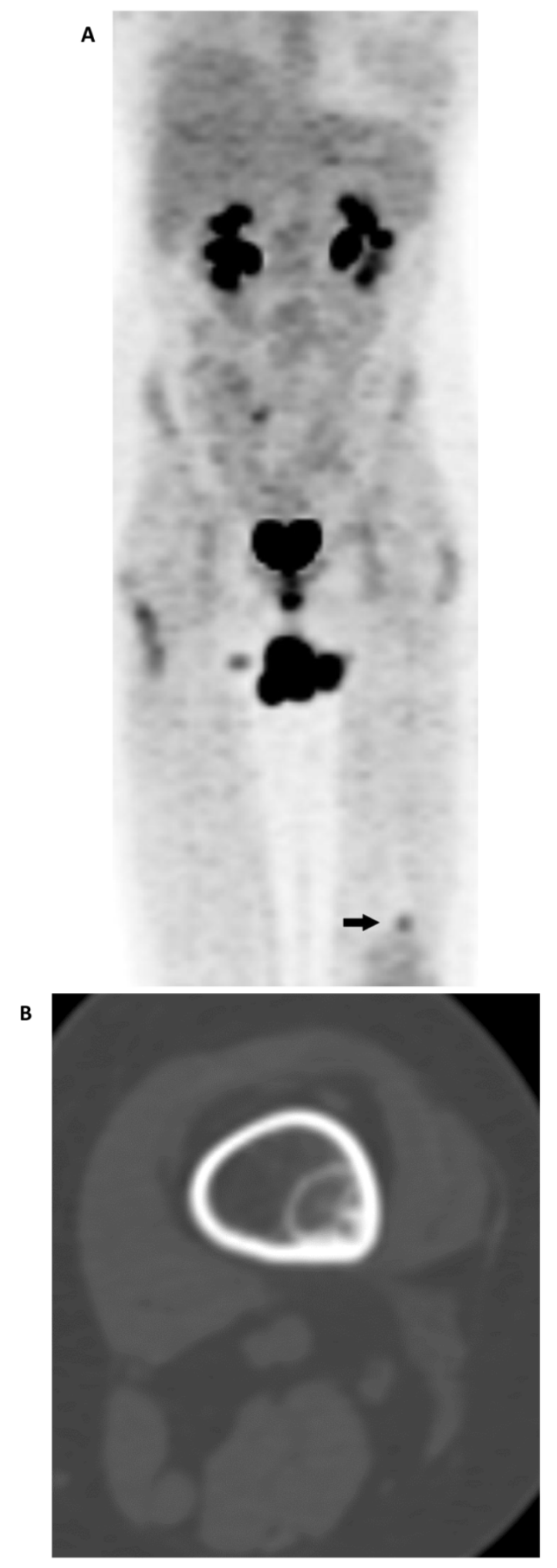

Fig 2. (A) Maximum intensity projection (MIP) PET-only image demonstrates a focal, FDG-avid lesion in the distal left femur (arrow). It is of concern in this patient with osteosarcoma of the sacrum (resected). Note is made of urinary incontinence accounting for the FDG accumulation in the perineal region. (B) CT-BW shows a cortically based lesion with a complete sclerotic rim and internal mineralization that is compatible with fibrous matrix. The anatomic appearance of the lesion is indicative of nonossifying fibroma (fibroxanthoma). 
A

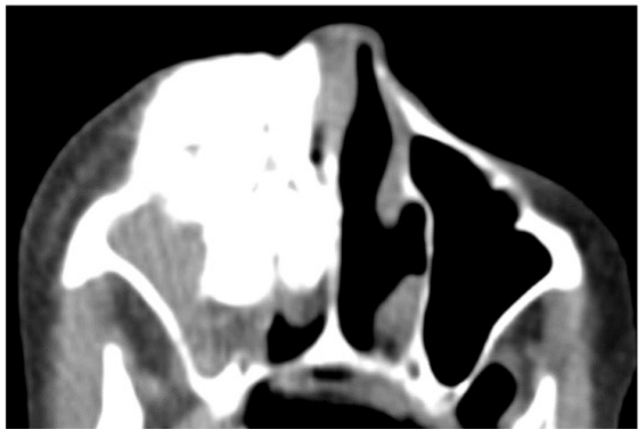

B

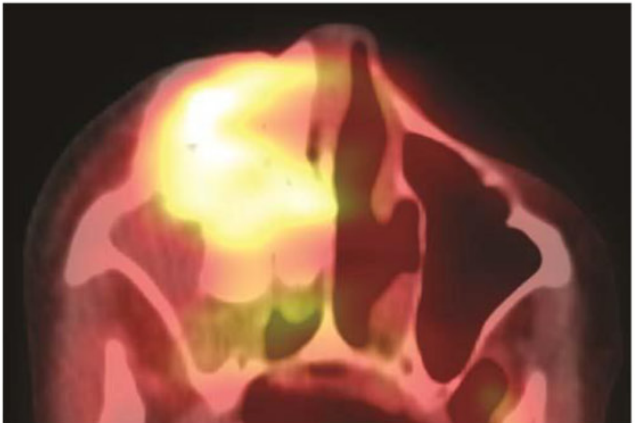

C

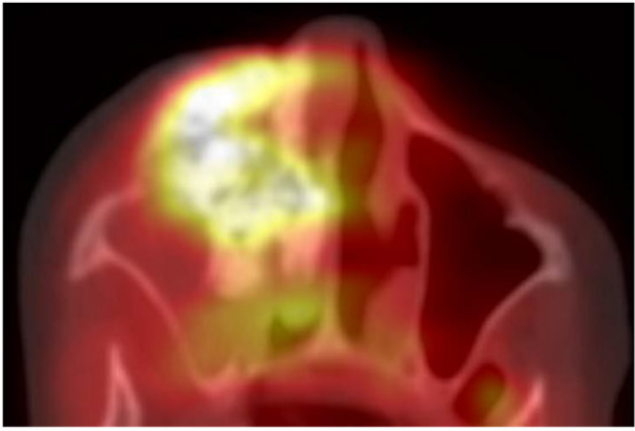

D

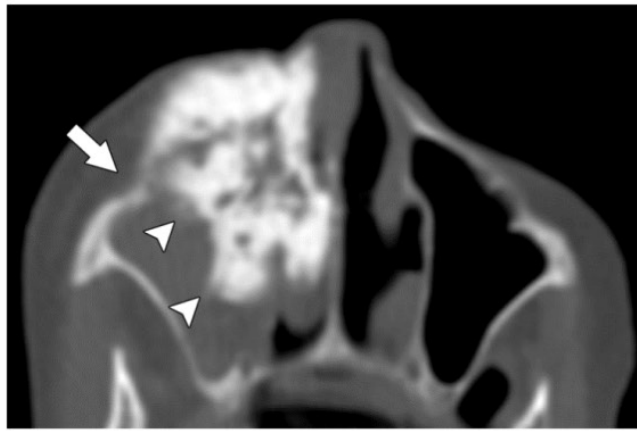

Fig 3. Tumor of the anterior and medial walls of the right maxilla in an 83-year-old woman. (A) CT-STW shows a dense tumor that appears well defined and smoothly lobulated. (B) FDG avidity demonstrated by the fused PET/CT-STW dataset raises the suspicion of malignancy (SUVmax 5.6). (C) While a greater degree of bone detail becomes apparent on the hybrid PET/CT-BW dataset, the tumor itself remains largely obscured by the extent of the high FDG uptake. Unlike the mildly FDG-avid Ewing sarcoma in Fig. 4D, the high avidity of most malignancies is likely to obscure the fine detail otherwise demonstrated by the bone windows. This may be the reason why no statistically significant relationships were found with the fused PET/CT-BW dataset. (D) Osteolysis is most readily apparent on CT-BW (arrow). III-defined margins are also now identified (arrowheads), further supporting the diagnosis of malignancy. The tumor contains a combination of centrally dense and peripherally "fluffy" mineralized osteoid matrix. Biopsy indicated osteosarcoma.
A

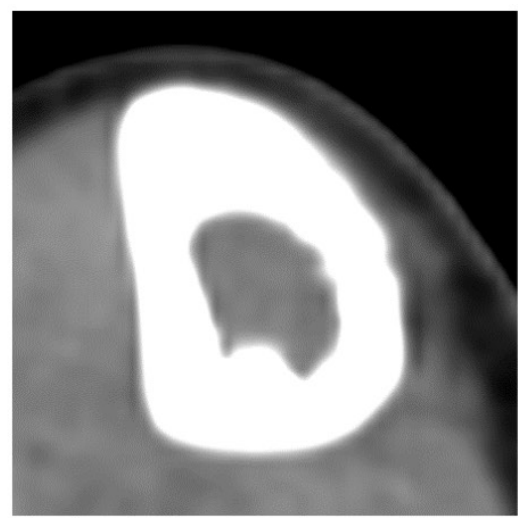

B

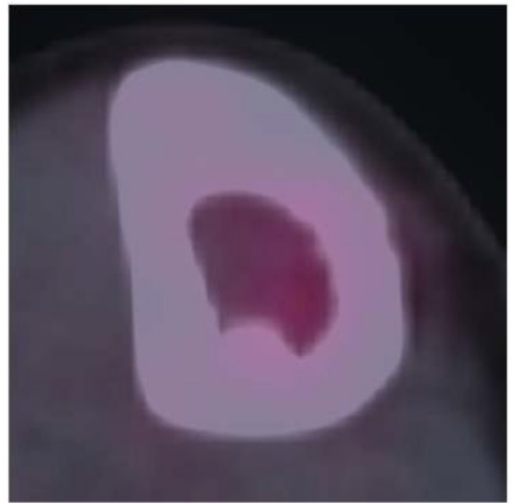

C

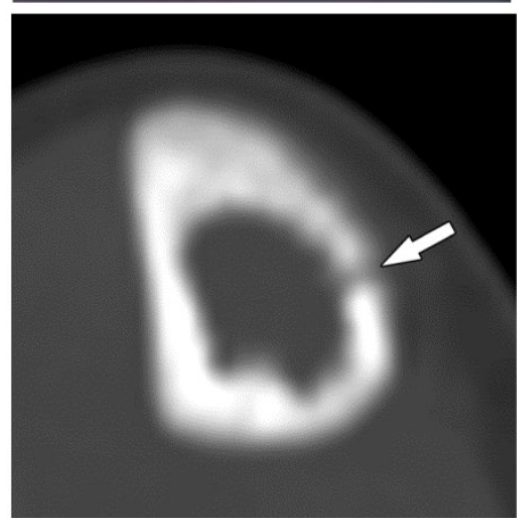

D

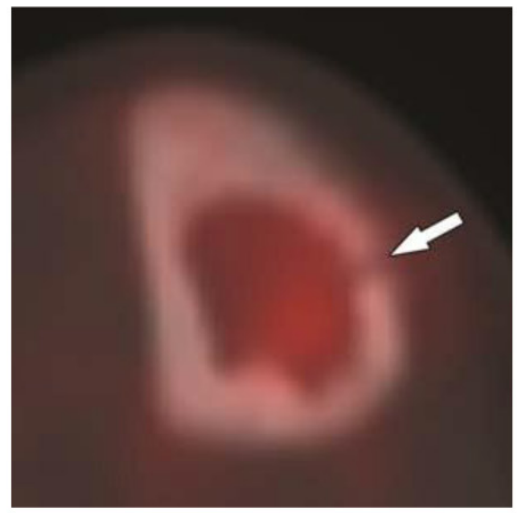

Fig 4. Tumor of the right tibial diaphysis in a 12-year-old girl. (A) A combination of intact- appearing cortex on CT-STW and (B), low FDG avidity on PET/CT-STW make the lesion appear non-aggressive with soft tissue windows. Utilizing bone windows, osteolysis is seen in the medial cortex on (C) CT-BW (arrow) and (D) PET/CT-BW (arrow), indicating the aggressive nature of the tumor. Biopsy indicated Ewing sarcoma. 


\section{Competing Interests}

No competing interests.

\section{References}

1. Becker H, Norman D, Boyd DP, Hattner RS, Newton TH. Computed tomography in detecting calvarial metastases: a comparison with skull radiography and radionuclide scanning. Neuroradiology. 1978; 16:504-505.

2. [Internet] University of South Carolina. Digital Imaging. http://radiology.med.sc.edu/6digimg.html.

3. Tecce PM, Fishman EK. Spiral CT with multiplanar reconstruction in the diagnosis of sternoclavicular osteomyelitis. Skeletal Radiol. 1995; 24(4):275-281.

4. Kashiwagi N, Hirabuki N, Andou K, Yoshifumi N, Tanaka H, Morino H, et al. MRI and CT findings of the giant cell tumors of the skull; five cases and a review of the literature. Eur J Radiol. 2006; 58(3):435-443.

5. Laval-Jeantet M, Paxton L, Frija J, Preteux F. Observer variation in bone lesion detection in thoraco-abdominal visceral CT images. Eur J Radiol. 1985; 5(4):310-312.

6. Pomerantz SM, White CS, Krebs TL, Daly B, Sukumar SA, Hooper F, et al. Liver and bone window settings for soft-copy interpretation of chest and abdominal CT. AJR Am J Roentgenol. 2000; 174(2):311-314.

7. Herrlin K, Ekelund L, Lovdahl R, Persson B. Computed tomography in suspected osteoid osteomas of tubular bones. Skeletal Radiol. 1982; 9(2):92-97.

8. Vandemark RM, Shpall EJ, Affronti ML. Bone metastases from breast cancer: value of CT bone windows. J Comput Assist Tomogr. 1992; 16(4):608-614.

9. Avila NA, Dwyer AJ, Rabel A, Darling T, Hong CH, Moss J. CT of sclerotic bone lesions: imaging features differentiating tuberous sclerosis complex with lymphangioleiomyomatosis from sporadic lymphangioleiomymatosis. Radiology. 2010; 254(3):851-857.

10. Salvati M, Ciappetta P, Raco A. Osteosarcomas of the skull. Clinical remarks on 19 cases. Cancer. 1993; 71(7):2210-2216.

11. Avrahami E, Even I. Osteoma of the inner table of the skull--CT diagnosis. Clin Radiol. 2000; 55(6):435-438.

12. Ryan JT, Haygood TM, Yamal JM, Evanoff M, O'Sullivan P, McEntee M, et al. The "memory effect" for repeated radiologic observations. AJR Am J Roentgenol. 2011; 197(6):W985-991.

13. Shankar LK, Hoffman JM, Bacharach S, Graham MM, Karp J, Lammertsma AA, et al. Consensus recommendations for the use of 18F-FDG PET as an indicator of therapeutic response in patients in National Cancer Institute Trials. J Nucl Med. 2006; 47(6):1059-1066.

14. Dehdashti F, Siegel BA, Griffeth LK, Fusselman MJ, Trask DD, McGuire $\mathrm{AH}$, et al. Benign versus malignant intraosseous lesions: discrimination by means of PET with 2-[F-18]fluoro-2-deoxy-D-glucose. Radiology. 1996; 200(1):243-247.

15. Aoki J, Watanabe H, Shinozaki T, Takagishi K, Ishijima H, Oya N, et al. FDG PET of primary benign and malignant bone tumors: standardized uptake value in 52 lesions. Radiology. 2001; 219(3):774-777.

16. Kole AC, Nieweg OE, Hoekstra HJ, van Horn JR, Koops HS, Vaalburg W. Fluorine-18-fluorodeoxyglucose assessment of glucose metabolism in bone tumors. J Nucl Med. 1998; 39(5):810-815.

17. Schulte M, Brecht-Krauss D, Heymer B, Guhlmann A, Hartwig E, Sarkar MR, et al. Grading of tumors and tumorlike lesions of bone: evaluation by FDG PET. J Nucl Med. 2000; 41(10):1695-1701.

18. Feldman F, Van Heertum R, Saxena C, Parisien M. 18FDG-PET applications for cartilage neoplasms. Skeletal Radiol. 2005; 34(7):367-374.

19. Strobel K, Exner UE, Stumpe KD, Hany TF, Bode B, Mende K, et al. The additional value of $\mathrm{CT}$ images interpretation in the differential diagnosis of benign vs. malignant primary bone lesions with 18F-FDG-PET/CT. Eur J Nucl Med Mol Imaging. 2008; 35(11):2000-2008.

20. Brenner W, Conrad EU, Eary JF. FDG PET imaging for grading and prediction of outcome in chondrosarcoma patients. Eur J Nucl Med Mol Imaging. 2004; 31(2):189-195.

21. Aoki J, Watanabe $H$, Shinozaki $T$, Tokunaga $M$, Inoue $T$, Endo K. FDG-PET in differential diagnosis and grading of chondrosarcomas. J Comput Assist Tomogr. 1999; 23(4):603-608.

22. Shin DS, Shon OJ, Han DS, Choi JH, Chun KA, Cho IH. The clinical efficacy of (18)F-FDG-PET/CT in benign and malignant musculoskeletal tumors. Annals of nuclear medicine. 2008; 22(7):603-609.

23. Charest M, Hickeson M, Lisbona R, Novales-Diaz JA, Derbekyan V, Turcotte RE. FDG PET/CT imaging in primary osseous and soft tissue sarcomas: a retrospective review of 212 cases. Eur J Nucl Med Mol Imaging. 2009; 36(12):1944-1951.

24. Costelloe CM, Madewell J. Radiography in the Initial Diagnosis of Primary Bone Tumors. AJR Am J Roentgenol. 2013; 200(1):3-7.

25. Madewell JE, Ragsdale BD, Sweet DE. Radiologic and pathologic analysis of solitary bone lesions. Part I: internal margins. Radiol Clin North Am. 1981; 19(4):715-748.

26. Costelloe CM, Rohren EM, Madewell JE, Hamaoka T, Theriault RL, Yu $\mathrm{TK}$, et al. Imaging bone metastases in breast cancer: techniques and recommendations for diagnosis. Lancet Oncol. 2009; 10(6):606-614. 